\title{
Code Translation
}

National Cancer Institute

\section{Source}

National Cancer Institute. Code Translation. NCI Thesaurus. Code C70898.

An automated procedure that uses a specialized set of concept descriptors for conversion of the target concept descriptors into executable elements of other code systems with the equivalent semantics. 